\title{
Robust Portfolio Optimization in an Illiquid Market in Discrete-Time
}

\author{
Nikolay Andreev \\ Financial Engineering and Risk Management laboratory, National Research University Higher School of \\ Economics, 20 Myasnitskaya Ulitsa, 101000 Moscow, Russia; nandreev@hse.ru
}

Received: 27 October 2019; Accepted: 21 November 2019; Published: 24 November 2019

\begin{abstract}
We present a robust dynamic programming approach to the general portfolio selection problem in the presence of transaction costs and trading limits. We formulate the problem as a dynamic infinite game against nature and obtain the corresponding Bellman-Isaacs equation. Under several additional assumptions, we get an alternative form of the equation, which is more feasible for a numerical solution. The framework covers a wide range of control problems, such as the estimation of the portfolio liquidation value, or portfolio selection in an adverse market. The results can be used in the presence of model errors, non-linear transaction costs and a price impact.
\end{abstract}

Keywords: dynamic games; robust optimization; dynamic programming; portfolio selection; transaction costs

MSC: 91A25; 91G10

JEL Classification: C61; C7; G11

\section{Introduction}

In this paper, we consider a robust approach to the constrained portfolio optimization problem in the presence of transaction costs and a price impact on the market. Optimal portfolio selection has become a popular topic in the mathematical finance literature since the pioneering works of Samuleson [1] and Merton [2]. Both papers consider a stochastic dynamic programming approach to the portfolio optimization problem on an efficient frictionless market with no trading limits. The continuous-time Samuelson model has been extended in various studies, see Reference [3] for an overview of the field. The works by Magill \& Constantinides [4] and Davis \& Norman [5], among others, paved the way for a new direction in research by considering a market with friction and introducing linear transaction costs, see Reference [6] for an overview. Portfolio selection for a discrete-time market, however, has not been given as much attention in the academic literature as its continuous-time counterpart, since in most cases it does not provide the Hamilton-Jacoby-Bellman equation (or quasi-variational inequality) to get the solution in a closed form, but requires a numerical solution instead. The discrete-time approach has been implemented in Bertsimas \& Lo [7] and Almgren $[8,9]$ for the optimal execution problem when an investor chooses the optimal strategy of the portfolio liquidation over a given number of consecutive periods.

The assumptions of the classic financial market model have undergone heavy criticism in view of the LTCM crisis in 1998 [10]. One of the main topics of discussion was the VAR risk metric, which assumed the Samuelson price model based on geometric Brownian motion and underestimated the risk of sudden price movements. Another question concerned the significance of market liquidity and transaction costs. Many works have been presented to deal with both problems. In References $[8,9,11]$, the authors introduce a trade-off between profit and volatility in a portfolio optimization framework in 
the presence of costs. Reference [12] accounts for the sudden price movements by introducing jumps into the price model. Nevertheless, most frameworks are still based on the arithmetic or the geometric Brownian motion model. In this paper, we present a discrete-time portfolio optimization framework in the presence of ambiguity in an illiquid market. The approach can be used to account for the impact of model error, which might be significant in many cases and especially during a financial crisis, when the investor needs a conservative estimate of the portfolio liquidation value, or when the portfolio needs to be rebalanced in a short period of time.

We adapt the framework of Smirnov $[13,14]$ and introduce a portfolio optimization problem as a game against nature where an investor tries to maximize the expected worst-case reward from a portfolio by making general and non-restrictive assumptions about the market. The robust optimization techniques have been introduced for a variety of economic problems recently. Deng et al. in Reference [15] combines the robust approach with the Markowitz framework to solve a one-period portfolio selection problem in a frictionless market, while Reference [16] solves a multi-stage asset allocation problem with linear costs by considering a series of linear programming problems with ambiguous constraints. In Reference [17], the authors present a game theoretic approach to the risk management control problem in the market with ambiguous coefficients of the Samuelson model. Robust optimization techniques have also been used to quantify the impact of the model error when assessing portfolio risks, see for example, Reference [18]. In this paper, we provide a framework for the multi-period portfolio optimization in the presence of model error by introducing the ambiguity in the market price distribution. We consider a general convex transaction costs function to account for the non-linear cost growth, and non-Markov dynamics of the system to account for a price impact.

The paper is structured as follows. In Section 1 we provide an overview of the problem. Section 2 provides a short overview of the research. In Section 3, we formulate the optimization framework, introduce the assumptions about the market, and present the corresponding Bellman-Isaacs equation. In Section 4, we study the sufficient conditions that allow the equation to be simplified, making it more feasible for a numerical solution. Section 5 discusses the results.

\section{Overview of the Main Results}

Consider an investor who tries to maximize the reward from the provided portfolio by the end of the investment horizon. Usually, the investor assumes some market price model, which allows them to estimate the distribution of the portfolio future wealth for any appropriate strategy, and make the correct investment decision. In this research, we assume that the investor does not possess the knowledge of the market price model. Instead, they have the estimates of the expected value and range of the future price movements. Both estimates are usually relevant for any investment process and can be interpreted in business terms. Below, we formulate the optimization problem in the presence of ambiguity and reduce it to the corresponding Bellman-Isaacs equation.

The rest of the research is devoted to simplifying the Bellman-Isaacs equation, since, in its original form, the equation requires estimating the worst expected value of the value function among all the appropriate candidate market models, each represented by the corresponding probability measure. Optimization over a set of measures does not provide an analytic solution in many practical cases, and the problem should be solved numerically. Nevertheless, some insight about the solution can be obtained in the most general case. In Theorem 2 below we show that the worst expected value is actually attained over a subset of atomic measures where the maximum number of atoms is defined by the dimension of the considered space. The result can be strengthened if (i) the value function remains concave with respect to the ambiguous parameters throughout the investment periods, and (ii) the problem can be formulated in terms of the position market values instead of volumes and market prices. In Theorem 4 we provide the corresponding sufficient conditions and prove that in this case the worst expected value is actually attained over a subset of atomic measures concentrated in the extreme points of the support. This allows to search for the optimal measure only on a subset of the support which might prove useful for a numerical implementation. 


\section{The Robust Portfolio Optimization Problem}

Consider a market with a finite horizon and discrete time $t=0,1, \ldots, N$. The proposed framework describes a consecutive process of decision-making by an investor who manages a portfolio trying to attain maximum reward at the end of the strategy. Each decision is made at the beginning of the investment period, while the outcome is observed at the end of the period. Throughout the paper, $t$ will denote the beginning of the corresponding period.

Assume that there are $n$ risky assets and one risk-free asset on the market. Let $X_{t}^{i}$ be the market price of the $i$-th risky asset at time $t$ and let $X_{t}=\left(X_{t}^{1}, \ldots, X_{t}^{n}\right)$. Let $Y_{t}$ be the market price of the risk-free asset. In the rest of the paper, the term "price" means the market price. The price of any risky asset at time $t$ might be undetermined at $t-1$ while the exact value of $Y_{t}$ is assumed to be known at $t-1$ (such property will be referred to as predictability later on). An example of a risk-free asset is the price of money in the portfolio's base currency. Another example is a sovereign bond account if the bonds are assumed risk-free and liquid, and if they are issued and traded in the portfolio's base currency. For the rest of the paper, we assume that all prices are positive. Note that, while this is a classic assumption that is reasonable in many practical cases, it does not fully account for the risk of bankruptcy of the issuer. We also do not account for the price discretization and assume that $X_{t}^{1}, \ldots, X_{t}^{n}, Y_{t} \in \mathbb{R}$.

By portfolio at time $t$ we mean a predictable vector of asset volumes $\hat{H}_{t}=\left(H_{t}^{1}, \ldots, H_{t}^{n}, H_{t}^{\gamma}\right)$. Predictability means that the portfolio structure for the next investment period is decided at the beginning of that period. We assume that all the assets are infinitely divisible: $H_{t}^{1}, \ldots, H_{t}^{n}$, $H_{t}^{Y} \in \mathbb{R}$. At the start of the strategy the investor is provided with the initial portfolio $\hat{H}_{0}$. Let $H_{t}=\left(H_{t}^{1}, \ldots, H_{t}^{n}\right) \in \mathbb{R}^{n}$ denote the volumes of the risky assets at $t$.

To account for the friction in the market, we assume that each trade by the investor incurs transaction costs. Costs can be categorized as explicit, such as broker commissions and fees, and implicit, which are due to the insufficient liquidity of the market: a trade moves the corresponding market price against it, therefore the average price of the trade might be worse than the market price if the volume is sufficiently large. Thus, in the frictionless market, a portfolio can be rebalanced with no loss of market value. In the real market, however, any rebalancing might lead to the loss of portfolio market value, which can be thought of as carrying additional costs. We assume that there are no costs incurred by the trades in the risk-free asset.

We assume that all information about the market is contained in the prices of the assets. The system state can therefore be represented as a combination of the price values and the portfolio. Let $W_{t}^{Y}=H_{t}^{Y} Y_{t}$ be the market value of the risk-free position at time $t$. Since the risk-free asset is assumed to be absolutely liquid, it is easier to operate in terms of $W_{t}^{Y}$ then work with $H_{t}^{Y}$ and $Y_{t}$ separately. Thus, by the system state $S_{t}$ at time $t$, we would mean a combination of $H_{t}, X_{t}$ and $W_{t}^{Y}$. We will also refer to the system history $S_{0}, \ldots, S_{t}$ up to the moment $t$ as $\bar{S}_{t}$. For the ease of notation, the functional dependence on the "." argument will mean the dependence on the system's past history throughout the paper. The dependence will sometimes be explicitly written out to clarify the dependence on the components of $\bar{S}_{t}$.

Let $C_{t}\left(\cdot, H_{t}\right)$ denote the costs carried when transitioning to the portfolio $H_{t}$. The function $C_{t}\left(\cdot, H_{t}\right)$ is assumed predictable. We do not introduce any additional inflows and outflows of assets, hence the following budget equation should hold for all $t$ :

$$
\Delta H_{t}^{Y} Y_{t-1}+\Delta H_{t} X_{t-1}=-C_{t}\left(\cdot, H_{t}\right)
$$

where $\Delta$ is the backward difference operator, that is, $\Delta H_{t}=H_{t}-H_{t-1}$. Both in Equation (1) and in the rest of the paper, the product of vectors should be understood as the inner product. Equation (1) allows us to express $H_{t}^{Y}$ as a function of $H_{t}$ and the past history as

$$
H_{t}^{Y}\left(\cdot, H_{t}\right)=Y_{t-1}^{-1}\left(W_{t-1}^{Y}-\left(H_{t}-H_{t-1}\right) X_{t-1}-C_{t}\left(\cdot, H_{t}\right)\right),
$$


thus the optimization problem will be stated in terms of $H_{t}$ instead of $\hat{H}_{t}$ in the rest of the paper, and by the portfolio strategy we mean the sequence of $H_{1}, \ldots, H_{N}$.

We assume that at each time $t$ the portfolio structure might be subject to the predictable constraints:

$$
H_{t} \in D_{t}(\cdot) \neq \varnothing \text {. }
$$

The constraint sets $D_{t}(\cdot)$ can be interpreted as the trading limits, which might serve the risk-management purposes or originate from the aims of the portfolio strategy. For example, consider a portfolio liquidation problem where an investor tries to close the risky positions over $N$ consecutive periods. The problem can be formulated in several ways, one of which is gradually reducing the allowed volumes of risky assets via the constraints, that is,

$$
D_{1}(\cdot) \supseteq D_{2}(\cdot) \supseteq \ldots \supseteq D_{N}(\cdot) .
$$

$D_{t}(\cdot)=\varnothing$ would have meant the inability to continue with the investment process and the early termination of the strategy. This case would have complicated the presentation of the results with technical details, so we assume the non-emptiness of $D_{t}(\cdot)$ throughout the paper. As for the other properties, from the risk-management point of view, it is usually reasonable to assume the boundedness of the constraint sets, since the unbounded sets might encourage entering infinitely large risky positions that are subject to infinite risk.

At $t-1$, the risk-free price $Y_{t}$ is assumed to be known by the investor. Consider the risk-free price dynamics in a multiplicative form as

$$
Y_{t}=R_{t} Y_{t-1}, \quad t=1, \ldots, N
$$

where $R_{t}>0$ is assumed predictable. $R_{t}-1$ can be interpreted as the risk-free rate of return. By combining (2) and (3), we can represent $W_{t}^{Y}$ as a function of portfolio $H_{t}$ and the past history as

$$
W_{t}^{Y}\left(\cdot, H_{t}\right)=H_{t}^{Y} Y_{t}=R_{t} H_{t}^{Y} Y_{t-1}=R_{t}\left(W_{t-1}^{Y}-\left(H_{t}-H_{t-1}\right) X_{t-1}-C_{t}\left(\cdot, H_{t}\right)\right) .
$$

As for the risky assets, the only assumption about the prices considers the range of returns. Let the risky price dynamics be represented in a multiplicative form as

$$
X_{t}^{i}=\xi_{t}^{i} X_{t-1}^{i}, \quad \xi_{t}^{i}>0, \quad i=1, \ldots, n, \quad t=1, \ldots, N,
$$

where $\xi_{t}^{i}-1$ is the return of the $i$-th asset over the investment period starting at $t-1$. Let $\xi_{t}=\left(\xi_{t}^{1}, \ldots, \xi_{t}^{n}\right) \in \mathbb{R}^{n}$ and let $\Lambda\left(\xi_{t}\right)$ be a diagonal matrix with $\xi_{t}$ on the main diagonal. Then we can write

$$
X_{t}=\Lambda\left(\xi_{t}\right) X_{t-1}, \quad t=1, \ldots, N
$$

The main assumption about market ambiguity is that we assume that $\xi_{t}$ belongs to a predefined set $K_{t}\left(S_{0}, \ldots, S_{t-1}\right) \subset \mathbb{R}^{n}$ for each $t=1, \ldots, N$, whereas the sets $K_{1}\left(\bar{S}_{0}\right), \ldots, K_{N}\left(\bar{S}_{N-1}\right)$ are assumed convex and compact.

Below, we present the notion of optimality and the Bellman-Isaacs equation. Classic portfolio selection frameworks usually assume that the investor maximizes the expected reward function of the portfolio at the end of the strategy. for example, in Reference [7], the authors consider the negated value of the execution costs as a reward, while in Reference [8], the mixture of the execution costs and the market risk is optimized. Many frameworks are based on expected utility maximization, where the form of the utility reflects the risk-aversion of the investor. Our approach accounts for both the uncertainty and the ambiguity of the market-the former refers to the stochastic nature of the market given a specific market model, while the latter refers to the uncertainty about the market model itself. In the presence of ambiguity, the standard expected von Neumann-Morgenstern utility cannot be used 
to define the optimal strategy, see the Ellsberg paradox [19]. Instead, we say that the portfolio strategy is optimal if it maximizes the robust Savage representation as defined in Reference [20] (see Theorem 2.80). For this, we assume the reasonable additional axioms of uncertainty aversion and certainty independence presented in Reference [20], when describing the behavior of the investor. The form of the robust Savage representation resembles the worst-case expected reward, which makes it a natural extension of the classic framework.

Remark 1. Optimization of the worst-case expected von Neumann-Morgenstern utility might still be appropriate when applied to a particular investment process. Assume, for example, that the utility function is defined by the risk-averse decision-maker based on the subjective estimates of the market. At the same time, the optimal strategy is obtained by another agent, the analyst, who tries to maximize the expected value of the provided utility in the worst case based on the analyst's independent estimate. Thus, from the analyst's point of view, the utility is an exogenous reward function which should be used in the framework.

We represent the ambiguity of the market via a set of probability measures describing price dynamics. For each $t=1, \ldots, N$, consider a set of probability measures $\mathcal{P}_{t}(\cdot)$, where each measure describes the uncertainty of risky prices over the corresponding period (in terms of the distribution of $\xi_{t}$, see (5)). Let $\mathcal{P}_{t, N}(\cdot)$ be the set of probability measures describing the risky prices across the investment periods starting at $t-1$ or later. Throughout the paper, we assume the following properties:

1. for each $t=1, \ldots, N$ and every $Q \in \mathcal{P}_{t}(\cdot)$, supp $Q \subseteq K_{t}(\cdot)$ and $K_{t}(\cdot)$ has compact and convex values;

2. $\quad$ for each $t=1, \ldots, N$ and every $Q \in \mathcal{P}_{t}(\cdot), \int \xi Q(d \xi)=E_{t}(\cdot)$, where $E_{t}(\cdot) \in K_{t}(\cdot)$;

3. (The Rectangularity assumption) for each admissible portfolio strategy,

$$
\mathcal{P}_{t, N}\left(\bar{S}_{t-1}\right)=\mathcal{P}_{t}\left(\bar{S}_{t-1}\right) \times \ldots \times \mathcal{P}_{N}\left(\bar{S}_{N-1}\right)
$$

Property 1 refers to the previously mentioned assumption about the price dynamics. The integral of the vector $\xi$ in Property 2 should be interpreted element-wise as $\int \xi^{i} Q(d \xi)=E_{t}^{i}(\cdot)$ for $i=1, \ldots, n$ and $E_{t}(\cdot)=\left(E_{t}^{1}(\cdot), \ldots, E_{t}^{n}(\cdot)\right)$. Property 2 means that at the beginning of each period the investor has certain expectations about the future price values, whereas $E(\cdot)$ is the expected value based on the investor's estimate. The assumption is not restrictive since, in many practical cases, the investment process is based on the estimate of future price movements, which is provided by the analyst. Besides, dropping the assumption would lead to the degeneration of the strategy in some practical cases, which will be illustrated at the end of the Section. Property 3 is a generalization of the Rectangularity assumption of References [21,22], that can be interpreted as the independence of market ambiguity in the future from the present market model. As an example of ambiguity that does not comply with (6), consider a single-asset market with the multiplicative dynamics (5) and $N=2$. For each $t$, let $K_{t}(\cdot) \equiv[d, u], 0<d<u$, and let $\xi_{t}$ be either uniformly distributed on $\left[d, \frac{u+d}{2}\right]$ with probability $p$ or uniformly distributed on $\left(\frac{u+d}{2}, u\right]$ with probability $1-p$, where $p \in[0,1]$ is unknown, which makes the market model ambiguous. Let $Q_{t, p}$ denote the corresponding probability measure at $t$. Then $\mathcal{P}_{t}=\left\{Q_{t, p}: p \in[0,1]\right\}$ for $t=1,2$, and

$$
\mathcal{P}_{1} \times \mathcal{P}_{2}=\left\{Q_{1, p} \times Q_{2, p^{\prime}}: p, p^{\prime} \in[0,1]\right\} .
$$

On the other hand,

$$
\mathcal{P}_{1,2}=\left\{Q_{1, p} \times Q_{2, p}: p \in[0,1]\right\},
$$

hence $\mathcal{P}_{1,2} \neq \mathcal{P}_{1} \times \mathcal{P}_{2}$. The Rectangularity assumption means that the investor assumes that the market is "replenished" at each period in terms of ambiguity, which is reasonable in the context of the worst-case framework. See Reference [22] for a similar example for the Ellsberg paradox. 
In this paper, we do not focus on the formal definition of the admissible strategies of the investor and the market, for a formal definition see for example, Reference [23]. Let $J\left(\bar{S}_{N}\right)$ be the reward function which depends on the portfolio structure and the market state. We assume that $J$ has values from $\mathbb{R} \cup\{-\infty\}$, where the $-\infty$ value means the failure of the investment strategy. Let $\mathcal{H}$ be the set of the admissible portfolio strategies, and let $\mathcal{H}_{t, N}(\cdot)$ be the set of the admissible strategies over the periods starting at $t-1$ or later. Let $\mathcal{P}=\mathcal{P}_{1, N}\left(S_{0}\right)$ be the set of probability measures describing the market dynamics across all periods. Then we can say that the portfolio strategy $H^{*} \in \mathcal{H}$ is optimal if

$$
\sup _{H \in \mathcal{H}} \inf _{Q \in \mathcal{P}} \int J\left(\bar{S}_{N}\right) d Q=\inf _{Q \in \mathcal{P}} \int J\left(\bar{S}_{N}^{*}\right) d Q
$$

where $\bar{S}_{N}^{*}$ is the system history for the strategy $H^{*}$. The supremum in (7) might not be achieved in general. However, it is usually the case in practice, if the problem is stated correctly, so throughout the paper we assume the existence of an optimal strategy. Note that $\inf _{Q \in \mathcal{P}} \int J(\cdot) d Q$ is the robust Savage representation we mentioned earlier.

Remark 2. Definition (7) requires integrability of the underlying reward function. In the presented practical framework, we do not focus on the question of measurability but assume the required form of measurability as needed. It will be shown in the next section, that we are mainly interested in the infimum over the subset of atomic measures from $\mathcal{P}_{t}(\cdot)$ that are concentrated in $n+1$ or fewer points of $K_{t}(\cdot), t=1, \ldots, N$, and every function is integrable with respect to any measure from that subset.

Let $V_{t-1}^{*}\left(\bar{S}_{t-1}\right)$ be the supremum of the robust Savage representation as estimated at time $t-1$, $t=1, \ldots, N$ :

$$
V_{t-1}^{*}\left(\bar{S}_{t-1}\right)=\sup _{\mathcal{H}_{t, N}\left(\bar{S}_{t-1}\right)} \inf _{Q_{t, N} \in \mathcal{P}_{t, N}\left(\bar{S}_{t-1}\right)} \int J\left(\bar{S}_{N}\right) d Q_{t, N}
$$

For $t=N$, let $V_{N}^{*}\left(\bar{S}_{N}\right) \equiv J\left(\bar{S}_{N}\right)$. We refer to $V_{t}^{*}$ as the "value function" in the rest of the paper. The proposed portfolio optimization problem can be solved via the dynamic programming principle which can be applied to the proposed framework:

Theorem 1. 1. The value function $V_{t}^{*}\left(\bar{S}_{t}\right)$ satisfies the following Bellman-Isaacs equation:

$$
\begin{gathered}
V_{t-1}^{*}\left(\bar{S}_{t-1}\right)=\sup _{H \in D_{t}\left(\bar{S}_{t-1}\right)} \inf _{Q \in \mathcal{P}_{t}\left(\bar{S}_{t-1}\right)} \int V_{t}^{*}\left(\bar{S}_{t}\right) d Q, \quad t=1, \ldots, N, \\
V_{N}^{*}\left(\bar{S}_{N}\right)=J\left(\bar{S}_{N}\right) .
\end{gathered}
$$

2. If for each $t=1, \ldots, N$ the supremum in (9) is achieved at some $H_{t}^{*}$, then $H^{*}=\left\{H_{1}^{*}, \ldots, H_{N}^{*}\right\}$ is an optimal strategy.

Proof. The proof is technical and has been moved to the Appendix A.

The dynamic programming principle makes the problem more feasible for the numerical solution by allowing us to reconstruct the value function for each investment period through an iterative process. By combining the formulae for the risk-free position value (4) and the price dynamics (5) with (9) and (10), we obtain the final form of the Bellman-Isaacs equation for the portfolio investment problem as

$$
\begin{gathered}
V_{t-1}^{*}\left(\bar{S}_{t-1}\right)=\sup _{H \in D_{t}\left(\bar{S}_{t-1}\right)} \inf _{Q \in \mathcal{P}_{t}\left(\bar{S}_{t-1}\right)} \int V_{t}^{*}\left(\bar{S}_{t-1}, H, \Lambda(\xi) X_{t-1}, W_{t}^{Y}\left(\bar{S}_{t-1}, H\right)\right) Q(d \xi), \\
V_{N}^{*}\left(\bar{S}_{N}\right)=J\left(\bar{S}_{N}\right) .
\end{gathered}
$$


The numerical solution of the provided equation might prove difficult due to the additional minimization problem in the recursive formula, which is not present in the classic stochastic framework. The problem is even more relevant due to the high dimension of the state space. In practical cases, the number of risky assets covered by the investment strategy can be quite high. For example, when the portfolio replicates a market index. In the next section, we provide sufficient conditions which allow the class of measures in the infimum problem to be narrowed down to atomic measures, which should make the Bellman-Isaacs equation more feasible for practical use.

Note that the value function $V_{t}^{*}(\cdot)$ might attain both $-\infty$ and $+\infty$ values. While the case $V_{t}^{*}(\cdot)=-\infty$ arises naturally given the definition of $J(\cdot), V_{t}^{*}(\cdot)=+\infty$ usually means an incorrect problem statement, since the possibility of infinite reward usually implies infinite risk of the optimal strategy. Therefore, in the rest of the paper we assume that $V_{t}^{*}(\cdot)<+\infty$ for each $t$. The assumption is satisfied for example, for the bounded reward function:

Proposition 1. If $J\left(\bar{S}_{N}\right) \leq$ a for some $a \in \mathbb{R}$, then $V_{t}^{*}\left(\bar{S}_{t}\right) \leq a$, for each $t=0, \ldots, N$.

Proof. We conduct the proof by backward induction. For $t=N$, the statement is true by definition of the value function. Assume that $V_{t}^{*}(\cdot) \leq a$ for some $t \leq N$. Then $\inf _{Q \in \mathcal{P}_{t}\left(\bar{S}_{t-1}\right)} \int V_{t}^{*}\left(\bar{S}_{t}\right) d Q \leq a$ and from the Bellman-Isaacs equation we get the statement for $V_{t-1}^{*}(\cdot)$.

If the reward function is unbounded, then proving that $V_{t}^{*}(\cdot)<+\infty$ would require some additional continuity assumptions, which is out of the scope of the presented research.

At the end of the current Section, we return to the question of the robust strategy in the absence of any expectations about risky prices, that is when $E_{t}(\cdot)$, as defined above, is also ambiguous. For example, consider a one period problem $(N=1)$ for a single risky asset $(n=1)$ with no costs $\left(C_{t}(\cdot, H) \equiv 0\right)$ and phase constraints of the form $D_{t}(\cdot)=[\underline{H}, \bar{H}]$, where $\underline{H}<0$ and $\bar{H}>0$, so that positive, negative and zero volumes are allowed. Let $R_{t} \in \operatorname{int} K_{t}(\cdot)$, which means that the risky price rate can either be higher or lower than the risk-free rate $R_{t}$ (the assumption is natural, since otherwise there would be obvious arbitrage in the market). Assume that $X_{t}=\xi_{t} X_{t-1}$, where the distribution of $\xi_{t}$ is ambiguous, and belongs to the family of distributions $\mathcal{P}_{t}$ with support $K_{t}(\cdot)$. Note that for $N=1$, the Rectangularity property is automatic. Assume that $\mathcal{P}_{t}$ contains all the Dirac measures, that is, the admissible strategies of the "market" include the deterministic strategies. Let the initial risky price be $X_{0}$ and let the initial portfolio have $H_{0}$ volume invested in the risky asset, while its risk-free position value is $W_{0}^{Y}$. Let the reward $J(\cdot)$ be a strictly monotone function of the portfolio market value $H_{N} X_{N}+W_{N}^{Y}$. For any admissible strategy $H$, the market value of the portfolio at $t=1$ would be

$$
W(H)=H X_{t}+R_{t}\left(W_{t-1}^{Y}-\left(H-H_{t-1}\right) X_{t-1}\right)=R_{t}\left(W_{t-1}^{Y}+H_{t-1} X_{t-1}\right)+\left(\xi-R_{t}\right) H X_{t-1},
$$

where $\xi \in K_{t}(\cdot)$. Note that if $H>0$ and the "market" chooses a deterministic strategy $\delta_{\xi^{*}}$, where $\xi^{*}<R_{t}$, then $W(H)<R_{t}\left(W_{t-1}^{Y}+H_{t-1}^{T} X_{t-1}\right)=W(0)$; similarly, if $H<0$, then we have $W(H)<W(0)$ for the deterministic "market" strategy $\delta_{\xi^{*}}$ with $\xi^{*}>R_{t}$. Note that $W(0)$ does not depend on the risky price (there are no risky investments in the portfolio). Therefore, the value of the robust Savage representation for any strategy $H \neq 0$ is less than its value for $H=0$, which makes $H=0$ the optimal strategy. The optimality of $H=0$ means that the investor should liquidate the risky positions completely. One can easily extrapolate the example to the multiperiod problem and see that $H=0$ is optimal for each period, which compromises the whole idea of the investment process. This example shows that to obtain a non-trivial strategy in this case, the investor needs to have some prior expectations about the future price values.

\section{Simplified Forms of the Bellman-Isaacs Equation}

This research provides a practical framework for the portfolio optimization problem. Therefore, we attempt to make economically reasonable assumptions while providing the interpreted solution via 
the Bellman-Isaacs equation. Still, a practical approach should allow for either an analytic or numerical solution. The Equations (11) and (12) does not allow for an analytic representation of the value function in most practical cases, while solving it numerically might be computationally difficult compared to the usual Bellman equation, since we need to solve an additional minimization problem at every step. Below, we consider particular cases of the framework that allow us to simplify the numerical solution of the internal minimization problem.

Let $\operatorname{ext}\left(K_{t}(\cdot)\right)$ be the set of extreme points of $K_{t}(\cdot)$. Let $\mathcal{P}_{t}^{n}(\cdot)$ (respectively, $\mathcal{P}_{t}^{n *}(\cdot)$ ) be the set of probability measures from $\mathcal{P}_{t}(\cdot)$ concentrated at $n+1$ or fewer points (respectively, extreme points) of $K_{t}(\cdot)$. Below we show that in some cases, the infimum over $\mathcal{P}_{t}$ in the Bellman-Isaacs equation is equivalent to the infimum over $\mathcal{P}_{t}^{n}$ or even $\mathcal{P}_{t}^{n *}$. For this, we use the following result:

Lemma 1. Let $f(x)$ be a real-valued Borel measurable function on convex and compact $K_{t}(\cdot) \subset \mathbb{R}^{n}$. Then (i) the infimum of $\int f(x) Q(d x)$ over the set of probability measures $\mathcal{P}_{t}(\cdot)$ equals the infimum over $\mathcal{P}_{t}^{n}(\cdot)$; (ii) if $f(x)$ is concave, the infimum over $\mathcal{P}_{t}(\cdot)$ coincides with the infimum over $\mathcal{P}_{t}^{n *}(\cdot)$.

Proof. The proof is technical and has been moved to the Appendix A. A similar result for a continuous $f(x)$ can be found in Smirnov [24]. The idea of their proof applies to our case as well. The proof of the Lemma 1 is based on their idea and is provided in this paper for consistency.

Lemma 1 immediately yields the following result, which allows us to narrow the set of measures in the Bellman-Isaacs equation:

Theorem 2. Assume that $V_{t}^{*}(\cdot)<+\infty$ and the sets $K_{t}(\cdot)$ are compact and convex for each $t=1, \ldots, N$. Then

$$
\begin{gathered}
V_{t-1}^{*}\left(\bar{S}_{t-1}\right)=\sup _{H \in D_{t}\left(\bar{S}_{t-1}\right)} \inf _{Q \in \mathcal{P}_{t}^{n}\left(\bar{S}_{t-1}\right)} \int V_{t}^{*}\left(\bar{S}_{t-1}, H, \Lambda(\xi) X_{t-1}, W_{t}^{Y}\left(\bar{S}_{t-1}, H\right)\right) Q(d \xi) \\
V_{N}^{*}\left(\bar{S}_{N}\right)=J\left(\bar{S}_{N}\right)
\end{gathered}
$$

Proof. Assume that for some $t, \bar{S}_{t-1}$ and $H, H \in D_{t}\left(\bar{S}_{t-1}\right)$, the subfunction $\xi \mapsto V_{t}^{*}\left(\bar{S}_{t-1}, H, \Lambda(\xi) X_{t-1}, W_{t}^{Y}\left(\bar{S}_{t-1}, H\right)\right)$ attains $-\infty$ for some $\xi^{\prime} \in K_{t}\left(\bar{S}_{t-1}\right), \xi^{\prime} \neq E_{t}\left(\bar{S}_{t-1}\right)$. Then the infimum is attained at the atomic measure concentrated at the points $\xi^{\prime}, \xi^{\prime \prime} \in K_{t}\left(\bar{S}_{t-1}\right)$, such that $E_{t}\left(\bar{S}_{t-1}\right)$ belongs to the interval connecting $\xi^{\prime}$ and $\xi^{\prime \prime}$. If $-\infty$ is attained exactly at $E_{t}\left(\bar{S}_{t-1}\right)$, then the corresponding atomic measure is concentrated at $E_{t}\left(\bar{S}_{t-1}\right)$. If $-\infty$ is not attained on $K_{t}\left(\bar{S}_{t-1}\right)$, then we use Lemma 1 to complete the proof.

Lemma 1 allows us to further narrow the class of measures when finding the infimum in the Bellman-Isaacs equation. For this, the subfunctions $\xi \mapsto V_{t}^{*}\left(\bar{S}_{t-1}, H, \Lambda(\xi) X_{t-1}, W_{t}^{Y}\left(\bar{S}_{t-1}, H\right)\right)$ in (11) should be concave. Below, we will prove the concavity via backward induction by imposing restrictions on the form of the cost function, the reward function, the constraint sets and the measure sets. Under the restrictions, the dependence on the risky position volumes $H$ would only be allowed through the dependence on the market values $H \circ X$, where "o" means the element-wise (Hadamard) product.

For further convenience, we sometimes represent the dependence on the system history $\bar{S}_{t}$ as the dependence on the arguments $\bar{S}_{t-1}, H_{t}, X_{t}$ and $W_{t}^{Y}$, thus decomposing the dependence on the latest system state and the history before that. We also uphold this notation for $t=0$, in which case the arguments should be interpreted as $H_{0}, X_{0}$ and $W_{0}^{Y}$, meaning that there is no history at the beginning of the strategy. We also sometimes represent the system history $\bar{S}_{t}$ as a combination of the history of the portfolio risky volumes $\bar{H}_{t}$, the history of the risk-free position value $\bar{W}_{t}^{Y}$ and the price history $\bar{X}_{t}$ up to time $t$. For example, $V_{t}^{*}\left(\bar{S}_{t}\right)$ might be equivalently written as $V_{t}^{*}\left(\bar{H}_{t}, \bar{X}_{t}, \bar{W}_{t}^{Y}\right)$ or $V_{t}^{*}\left(\bar{H}_{t-1}, \bar{X}_{t-1}, \bar{W}_{t-1}^{Y}, H_{t}, X_{t}, W_{t}^{Y}\right)$, whenever the notation is clear from the context. In the following 
proofs, we also make some monotonicity assumptions, in which case the comparison of vectors and arrays of arguments should be understood element-wise.

Assume that the constraint sets $D_{t}$ are monotonic in the following sense: let $d_{t}\left(\bar{W}_{t-1}^{Y}\right)$ be any of the subfunctions $\bar{W}_{t-1}^{Y} \mapsto D_{t}\left(\bar{S}_{t-1}\right)$, then

$$
d_{t}\left(\bar{W}_{t-1}^{Y, 1}\right) \subseteq d_{t}\left(\bar{W}_{t-1}^{Y, 2}\right) \quad \text { if } \quad \bar{W}_{t-1}^{Y, 1} \leq \bar{W}_{t-1}^{Y, 2} .
$$

The assumption means that the increase in the portfolio risk-free position should provide additional trading options while keeping the previous ones available. The assumption might be inappropriate in some cases, for example, when the increase in risk-free funds leads to additional constraints on the less risky positions in favor of the more risky ones to stimulate the more risky and profitable strategies. We do not consider such cases, believing that the investor is reasonable enough to account for the increased risk-free reserves when choosing the strategy, thus the additional trading limits are not required.

Assume that the measure sets $\mathcal{P}_{t}$ are monotonic in the following sense: let $p_{t}\left(\bar{W}_{t-1}^{Y}\right)$ be any of the subfunctions $\bar{W}_{t-1}^{Y} \mapsto \mathcal{P}_{t}\left(\bar{S}_{t-1}\right)$, then

$$
p_{t}\left(\bar{W}_{t-1}^{Y, 1}\right) \supseteq p_{t}\left(\bar{W}_{t-1}^{Y, 2}\right) \quad \text { if } \quad \bar{W}_{t-1}^{Y, 1} \leq \bar{W}_{t-1}^{Y, 2} .
$$

The assumption means that the increase in the risk-free funds does not lead to more ambiguity in the market, according to the investor's subjective estimate. Otherwise, we would have assumed that the investor becomes more uncertain when provided with the additional risk-free liquid assets, which is unreasonable.

Below, we provide sufficient conditions for the concavity and monotonicity of the value function.

Theorem 3. Let $V_{t}^{*}(\cdot)$ be the value function in the Bellman-Isaacs Equations (11) and (12). Assume that for each $t=1, \ldots, N$

- the subfunctions $\bar{H}_{t}^{Y}, \bar{W}_{t-1}^{Y} \mapsto C_{t}\left(\bar{S}_{t-1}, H_{t}\right)$ are convex;

- the subfunctions $\bar{W}_{t-1}^{Y} \stackrel{\longrightarrow}{\longmapsto} C_{t}\left(\bar{S}_{t-1}, H_{t}\right)$ are non-increasing;

- the subfunctions $\bar{H}_{N}, \bar{W}_{N}^{Y} \mapsto J\left(\bar{S}_{N}\right)$ are concave;

- $\quad$ the subfunctions $\bar{W}_{N}^{Y} \mapsto J\left(\bar{S}_{N}\right)$ are non-decreasing;

- $\quad$ the constraint sets $D_{t}$ have convex values and monotone in the sense of (15);

- $\quad$ the measure sets $\mathcal{P}_{t}$ are monotone in the sense of (16);

- $V_{t}^{*}\left(\bar{S}_{t}\right)<+\infty$.

Then the subfunctions $\bar{H}_{t}, \bar{W}_{t}^{Y} \mapsto V_{t}^{*}\left(\bar{S}_{t}\right)$ are concave and the subfunctions $\bar{W}_{t}^{Y} \mapsto V_{t}^{*}\left(\bar{S}_{t}\right)$ are non-decreasing for $t=1, \ldots, N$.

Proof. The proof is technical and has been moved to the Appendix A.

The convexity of the cost function is a common requirement in financial literature, which is justified in most practical cases due to the well-known price impact effect in the market. The assumption that the costs are non-increasing with respect to the risk-free position value is economically reasonable, since the additional risk-free and liquid funds should create more favorable conditions when choosing the execution strategy, while incurring no additional costs themselves. The concavity of the reward function is reasonable if the investor is risk-averse, while the monotonicity with respect to the risk-free position value is natural for many practical cases, when adding to the total portfolio value at no additional costs should increase the portfolio reward.

Let $W_{t}^{X}=H_{t} \circ X_{t}$ be the vector of risky position values at time $t$, and let $\bar{W}_{t}^{X}$ be the corresponding history up to time $t, t=0, \ldots, N$. Let $S_{t}^{W}=\left(W_{t}^{X}, W_{t}^{Y}\right)$ be the state of the portfolio in terms of the position market values. $S_{t}^{W}$ can be derived from $S_{t}$ and combines the market and portfolio states. Let $\bar{S}_{t}^{W}$ be the corresponding state history up to time $t$. Next, we show how the class of measures in the Bellman-Isaacs equation can be narrowed, if the value function can be represented as a function of $\bar{S}_{t}^{W}$. 
Theorem 4. Assume that all the assumptions of Theorem 3 hold and, in addition,

- the cost function has the following form:

$$
C_{t}\left(\bar{S}_{t-1}, H_{t}\right) \equiv \tilde{C}_{t}\left(\bar{S}_{t-1}^{W}, H_{t} \circ X_{t-1}\right)
$$

- the reward function has the following form:

$$
J\left(\bar{S}_{N}\right) \equiv \tilde{J}\left(\bar{S}_{N}^{W}\right)
$$

- the constraint sets $D_{t}(\cdot)$ are given in the form of constraints on the risky position market values:

$$
H \in D_{t}\left(\bar{S}_{t-1}\right) \quad \Leftrightarrow \quad H \circ X_{t-1} \in \tilde{D}_{t}\left(\bar{S}_{t-1}^{W}\right) ;
$$

- the probability measure sets $\mathcal{P}_{t}(\cdot)$ have the following form:

$$
\mathcal{P}_{t}\left(\bar{S}_{t-1}\right) \equiv \tilde{\mathcal{P}}_{t}\left(\bar{S}_{t-1}^{W}\right)
$$

Then

$$
\begin{gathered}
V_{t-1}^{*}\left(\bar{S}_{t-1}\right)=\sup _{H \in D_{t}\left(\bar{S}_{t-1}\right)} \inf _{Q \in \mathcal{P}_{t}^{n *}\left(\bar{S}_{t-1}\right)} \int V_{t}^{*}\left(\bar{S}_{t-1}, H, \Lambda(\xi) X_{t-1}, W_{t}^{Y}\left(\bar{S}_{t-1}, H\right)\right) Q(d \xi), \\
V_{N}^{*}\left(\bar{S}_{N}\right)=J\left(\bar{S}_{N}\right) .
\end{gathered}
$$

Proof. The statement can be proven by using Lemma 1, so we only need to prove the concavity of the subfunctions $\xi \mapsto V_{t}^{*}\left(\bar{S}_{t-1}, H, \Lambda(\xi) X_{t}, W_{t}^{Y}\left(\bar{S}_{t-1}, H\right)\right)$ for each $t=1, \ldots, N$. First, we will prove by backward induction that the value function $V_{t}^{*}$ can be represented in the form

$$
V_{t}^{*}\left(\bar{S}_{t}\right) \equiv \tilde{V}_{t}^{*}\left(\bar{S}_{t}^{W}\right)
$$

The statement holds for $t=N$ due to (18). Assume that it is true for some $t \leq N$. From (17), we get that $W_{t}^{Y}\left(\bar{S}_{t-1}, H_{t}\right)$ can be represented as a function of $\bar{S}_{t-1}^{W}$ and $H_{t} \circ X_{t-1}$. Indeed, from (4) we have

$$
\begin{gathered}
W_{t}^{Y}\left(\bar{S}_{t-1}, H_{t}\right)=R_{t}\left(W_{t-1}^{Y}-H_{t} X_{t-1}+H_{t-1} X_{t-1}-C_{t}\left(\bar{S}_{t-1}, H_{t}\right)\right) \\
=R_{t}\left(W_{t-1}^{Y}-\mathbf{1} \cdot\left(H_{t} \circ X_{t-1}\right)+\mathbf{1} \cdot\left(H_{t-1} \circ X_{t-1}\right)-\tilde{C}_{t}\left(\bar{S}_{t-1}^{W}, H_{t} \circ X_{t-1}\right)\right) \equiv \tilde{W}_{t}^{Y}\left(\bar{S}_{t-1}^{W}, H \circ X_{t-1}\right),
\end{gathered}
$$

where 1 means the vector $(1,1, \ldots, 1) \in \mathbb{R}^{n}$. Since $\Lambda(\xi)$ is a diagonal matrix, (23) implies that

$$
\begin{gathered}
V_{t}^{*}\left(\bar{S}_{t-1}, H, \Lambda(\xi) X_{t-1}, W_{t}^{Y}\left(\bar{S}_{t-1}, H\right)\right) \equiv \tilde{V}_{t}^{*}\left(\bar{S}_{t-1}^{W}, H \circ \Lambda(\xi) X_{t-1}, \tilde{W}_{t}^{Y}\left(\bar{S}_{t-1}^{W}, H \circ X_{t-1}\right)\right) \\
\equiv \tilde{V}_{t}^{*}\left(\bar{S}_{t-1}^{W}, \Lambda(\xi)\left(H \circ X_{t-1}\right), \tilde{W}_{t}^{Y}\left(\bar{S}_{t-1}^{W}, H \circ X_{t-1}\right)\right) .
\end{gathered}
$$

Therefore, $V_{t}^{*}\left(\bar{S}_{t-1}, H, \Lambda(\xi) X_{t-1}, W_{t}^{Y}\left(\bar{S}_{t-1}, H\right)\right)$ is, in fact, a function of $\bar{S}_{t-1}^{W}, H \circ X_{t-1}$ and $\xi$, which we will denote as $\phi_{t}\left(\bar{S}_{t-1}^{W}, H \circ X_{t-1}, \xi\right)$. Then, by substituting (19) and (20) into the Bellman-Isaacs equation, we get that

$$
V_{t-1}^{*}\left(\bar{S}_{t-1}\right)=\sup _{H \circ X_{t-1} \in \tilde{D}_{t}\left(\bar{S}_{t-1}^{W}\right)} \inf _{\tilde{\mathcal{P}}_{t}\left(\bar{S}_{t-1}^{W}\right)} \int \phi_{t}\left(\bar{S}_{t-1}^{W}, H \circ X_{t-1}, \xi\right) Q(\xi) .
$$


Therefore, $V_{t-1}^{*}\left(\bar{S}_{t-1}\right)$ is a function of $\bar{S}_{t-1}^{W}$, which proves the induction step, thus proving that the value function admits the representation (23) for $t=0, \ldots, N$. Then, from (23) and the diagonality of $\Lambda(\xi)$, we get that, for any $\xi \in K_{t}\left(\bar{S}_{t-1}\right)$,

$$
\begin{aligned}
V_{t}^{*}\left(\bar{S}_{t-1}, H, \Lambda(\xi) X_{t-1}, W_{t}^{Y}\right) & =\tilde{V}_{t}^{*}\left(\bar{S}_{t-1}^{W}, H \circ \Lambda(\xi) X_{t-1}, W_{t}^{Y}\right) \\
& =\tilde{V}_{t}^{*}\left(\bar{S}_{t-1}^{W},(\Lambda(\xi) H) \circ X_{t-1}, W_{t}^{Y}\right)=V_{t}^{*}\left(\bar{S}_{t-1}, \Lambda(\xi) H, X_{t-1}, W_{t}^{Y}\right) .
\end{aligned}
$$

By Theorem 3, the subfunctions $H \mapsto V_{t}^{*}\left(\bar{S}_{t-1}, H, X_{t}, W_{t}^{Y}\right)$ are concave. Therefore, the subfunctions $\xi \mapsto V_{t}^{*}\left(\bar{S}_{t-1}, \Lambda(\xi) H, X_{t}, W_{t}^{Y}\right)$ are concave as compositions of the linear function $\xi \mapsto \Lambda(\xi) H$ and the concave function. Then, (25) implies that the subfunctions $\xi \mapsto V_{t}^{*}\left(\bar{S}_{t-1}, H, \Lambda(\xi) X_{t}, W_{t}^{Y}\right)$ are concave as well, which means the concavity of the subfunctions $\xi \mapsto V_{t}^{*}\left(\bar{S}_{t-1}, H, \Lambda(\xi) X_{t}, W_{t}^{Y}\left(\bar{S}_{t-1}, H\right)\right)$ and proves the statement.

The conditions of Theorem 4 might seem too restrictive. However, they are satisfied in a variety of practical cases. Below, we provide some examples covered by the Theorem.

The form of the cost function (17) includes the widely-used affine model of costs, see Reference [5]. At the same time, it allows us to introduce the non-linear cost growth, which might be relevant for large portfolios.

As the example of the concave and monotonic reward function of the form (18), consider a utility function which depends on the portfolio value, for example,

$$
\tilde{J}\left(\bar{S}_{N}^{W}\right)=U\left(H_{N} X_{N}+W_{N}^{Y}\right)
$$

or

$$
\tilde{J}\left(\bar{S}_{N}^{W}\right)=U\left(H_{N} X_{N}+W_{N}^{Y}-C_{N+1}\left(\bar{S}_{N}^{W}\right)\right),
$$

where $U$ is a concave non-decreasing utility function and $C_{N+1}\left(\bar{S}_{N}\right)$ is the costs of liquidating the remaining risky assets in the portfolio after the end of the strategy (assume that $\bar{H}_{N}, \bar{W}_{N}^{Y} \mapsto C_{N+1}\left(\bar{S}_{N}\right)$ are convex and $\bar{W}_{N}^{Y} \mapsto C_{N+1}\left(\bar{S}_{N}\right)$ are non-increasing). In the first case, the investor wants to maximize the market value of the portfolio, and in the latter case, the liquidation value of the portfolio is being maximized.

As the example of the constraint sets covered by Theorem 4, consider a set of predictable functions $g_{t}^{i}(\cdot, x)$ for $t=1, \ldots, N$ and $i=1, \ldots, M$, such that the corresponding subfunctions $x \mapsto g_{t}^{i}(\cdot, x)$ are quasi-convex. Let $\beta_{t}^{i}(\cdot) \geq 0$ be predictable coefficients and let $W_{t-1}=H_{t-1} X_{t-1}+W_{t-1}^{Y}$ be the market value of the portfolio at $t-1$. Consider the constraint sets of the form:

$$
D_{t}(\cdot)=\left\{H: g_{t}^{i}\left(\cdot, H \circ X_{t-1}\right) \leq \beta_{t}^{i}(\cdot) W_{t-1}, i=1, \ldots, M\right\} .
$$

Quasi-convexity of $g_{t}^{i}$ implies the convexity of $D_{t}(\cdot)$ values, while non-negativity of $\beta_{t}^{i}(\cdot)$ implies the monotonicity as required by Theorem 4 . The introduced form of constraints includes several important trading limit types, for example:

1. $g_{t}^{i}\left(\cdot, H \circ X_{t-1}\right)=H^{i} X_{t-1}^{i}, i=1, \ldots, n$, means the constraints on the maximum value of each risky position in terms of the percentage of the portfolio market value;

2. $g_{t}^{i}\left(\cdot, H \circ X_{t-1}\right)=-H^{i} X_{t-1}^{i}, i=1, \ldots, n$, means the constraints on the minimum value of each risky position, which limits short-selling;

3. $g_{t}^{i}\left(\cdot, H \circ X_{t-1}\right)=\sum_{j=1}^{n} a_{t}^{i, j}(\cdot) H^{j} X_{t-1}^{j}$ means the constraints on the combinations of asset positions, which is useful for limiting investments in a group of products, which, for example, represent a sector of economy or constitute an index; a special case of this type of constraints is $g_{t}^{i}\left(\cdot, H \circ X_{t-1}\right)=\sum_{j=1}^{n} H^{j} X_{t-1}^{j}$, which might be used to limit the risk-free asset short-selling. 
Theorem 4 covers several price impact effects. For example, consider the measure sets $\mathcal{P}_{t}$ with the expected value $E_{t}(\cdot)=\left(E_{t}^{1}(\cdot), \ldots, E_{t}^{n}(\cdot)\right)$ defined as

$$
\ln E_{t}^{i}(\cdot)=\sum_{k=1}^{t-1} \mu_{k}^{i}+\sum_{k=1}^{t-1} \lambda_{k}^{i}\left(\Delta H_{k} \circ X_{k}\right)+\sum_{k=1}^{t-1} \gamma_{k}^{i}\left(\Delta H_{k} \circ X_{k}\right) e^{-\rho_{k}^{i}(t-k)},
$$

where $\mu_{k^{\prime}}^{i} \lambda_{k}^{i}(w), \gamma_{k}^{i}(w)$ and $\rho_{k}^{i}$ are assumed to be known, $i=1, \ldots, n$. For the $i$-th risky asset and time $k, \mu_{k}^{i}$ is the expected drift of the market price, $\lambda_{k}^{i}(w)$ is the expected permanent price impact of the trade with market value $w, \gamma_{k}^{i}(w)$ is the expected temporary price impact of the trade with market value $w$, and $\rho_{k}^{i}$ characterizes the expected resilience rate of the market price. The logarithm in the formula guarantees that the expected value of the multiplicative coefficient of the price dynamics is positive. Let the support of the measure set be defined as

$$
K_{t}(\cdot)=\left\{E_{t}(\cdot)\right\}+M_{t}
$$

where the " + " operator means the Minkowski sum, and $M_{t}$ characterizes both the uncertainty and the ambiguity of the market prices. Assume that $0 \in \operatorname{int} M_{t}$ to guarantee that $E_{t}(\cdot) \in K_{t}(\cdot), t=1, \ldots, N$. The provided forms of $E_{t}(\cdot)$ and $K_{t}(\cdot)$ capture several aspects of the price impact which are relevant for portfolio optimization in a market with limited liquidity, see for example, References $[8,11,25,26]$. The structure of $M_{t}$ characterizes the investor's estimate of the market ambiguity. For example, the investor might assume the rectangular structure to disregard any prior knowledge about the dependencies between the asset prices. On the other hand, by considering the elliptical form of $M_{t}$, the investor assumes that the prices will not attain the respective extreme values all at the same time, thus introducing some prior knowledge about the market into the framework.

\section{Conclusions}

In this paper, we present a robust approach to a general portfolio optimization problem. We introduce the ambiguity in the market by formulating the problem as a game against nature, where the investor attempts to maximize the worst-case reward from the portfolio at the end of the strategy. The robust approach considers the model error when selecting an optimal portfolio strategy, which is relevant when the parameters of the model cannot be reliably estimated, or when the model-based forecasts are untrustworthy, for example, during a financial crisis. The framework might be used as a decision support system when comparing portfolio strategies based on the model-independent estimates about the future market price values. Our goal is to present a practical approach, therefore all the assumptions are economically reasonable and well-interpreted.

Calculation of the value function in the Bellman-Isaacs Equations (11) and (12) involves a complex problem of optimizing over a set of distributions with the common mean and support. A direct solution of the problem would have required some approximation and parametrization of the measure set which would have raised the question of approximation error. Theorem 2 shows that such approximation is unnecessary and the problem can be reduced to optimization over a finite set of points of the support, thus can be solved numerically similar to the classic Bellman equation. Indeed, given the $n+1$ atoms of the distribution in $\mathbb{R}^{n}$, the probabilities of the atoms can be calculated directly as the corresponding barycentric coordinates of the distribution mean. Due to the recursive nature of the Equation (11), a derivative-free method, for example, a random search method, might be more appropriate for estimating the value of the robust Savage representation $\inf _{Q \in \mathcal{P}_{t}\left(\bar{S}_{t-1}\right)} \int V_{t}^{*}\left(\bar{S}_{t}\right) d Q$, and for reconstructing the value function. Theorem 4 states that under some assumptions, the search space for the method can be reduced, since the atoms of the optimal measure are guaranteed to be the extreme points of the support.

The research covers a wide range of problem statements which might include non-linear transaction costs and price impact, and various forms of trading limits. The provided framework 
can be applied for such classic portfolio optimization problems as the optimal execution of trades and the portfolio selection, and might be used as an analytic tool for the strategy evaluation and risk-management purposes in an ambiguous market.

Funding: Support from the Basic Research Program of the National Research University Higher School of Economics is gratefully acknowledged.

Acknowledgments: The author would like to thank Sergey Smirnov, Faculty of Computational Mathematics and Cybernetics, Lomonosov Moscow State University, for helpful ideas and insight.

Conflicts of Interest: The author declares no conflict of interest. The funders had no role in the design of the study; in the collection, analyses, or interpretation of data; in the writing of the manuscript, or in the decision to publish the results.

\section{Appendix A}

\section{Proof of Theorem 1}

Proof. We begin with the proof of the first part of the Theorem. Equations (9) and (10) for $t=N$ are true by definition of the value function, thus we only need to prove (9) for $t=1, \ldots, N-1$ in case $N>1$. For any $t=1, \ldots, N-1$ and any tail strategy $h_{t, N} \in \mathcal{H}_{t, N}\left(\bar{S}_{t-1}\right)$ over the periods starting at $t-1$ or later, let

$$
V_{t-1}\left(\bar{S}_{t-1}, h_{t, N}\right)=\inf _{Q_{t, N} \in \mathcal{P}_{t, N}\left(\bar{S}_{t-1}\right)} \int J\left(\bar{S}_{N}\right) d Q_{t, N},
$$

where $\bar{S}_{N}$ is the history for strategy $h_{t, N}$. Let $H_{t}$ be the part of strategy $h_{t, N}$ which covers the period starting at $t-1$, and let $h_{t+1, N}$ be the rest of $h_{t, N}$. Then for $t<N$, due to the Rectangularity property of $\mathcal{P}_{t, N}$, we have

$$
\begin{gathered}
V_{t-1}\left(\bar{S}_{t-1}, h_{t, N}\right)=\inf _{Q_{t, N} \in \mathcal{P}_{t, N}\left(\bar{S}_{t-1}\right)} \int J\left(\bar{S}_{N}\right) d Q_{t, N} \\
=\inf _{Q \in \mathcal{P}_{t}\left(\bar{S}_{t-1}\right)} \int d Q \inf _{Q_{t+1, N} \in \mathcal{P}_{t+1, N}\left(\bar{S}_{t}\right)} \int J\left(\bar{S}_{N}\right) d Q_{t+1, N}=\inf _{Q \in \mathcal{P}_{t}\left(\bar{S}_{t-1}\right)} \int V_{t}\left(\bar{S}_{t}, h_{t+1, N}\right) d Q .
\end{gathered}
$$

By definition (8), $V_{t-1}^{*}\left(\bar{S}_{t-1}\right)=\sup _{h_{t, N} \in \mathcal{H}_{t, N}} V_{t-1}\left(\bar{S}_{t-1}, h_{t, N}\right)$. Therefore,

$$
\begin{gathered}
V_{t-1}^{*}\left(\bar{S}_{t-1}\right)=\sup _{h_{t, N} \in \mathcal{H}_{t, N}\left(\bar{S}_{t-1}\right)} \inf _{Q \in \mathcal{P}_{t}\left(\bar{S}_{t-1}\right)} \int V_{t}\left(\bar{S}_{t}, h_{t+1, N}\right) d Q \\
\leq \sup _{h_{t, N} \in \mathcal{H}_{t, N}\left(\bar{S}_{t-1}\right)} \inf _{Q \in \mathcal{P}_{t}\left(\bar{S}_{t-1}\right)} \int V_{t}^{*}\left(\bar{S}_{t}\right) d Q=\sup _{H_{t} \in D_{t}\left(\bar{S}_{t-1}\right)} \inf _{Q \in \mathcal{P}_{t}\left(\bar{S}_{t-1}\right)} \int V_{t}^{*}\left(\bar{S}_{t}\right) d Q,
\end{gathered}
$$

because $V_{t}^{*}\left(\bar{S}_{t}\right)$ does not depend on $h_{t+1, N}$. On the other hand, since $V_{t}^{*}\left(\bar{S}_{t}\right)$ is the supremum of $V_{t}\left(\bar{S}_{t}, h_{t+1, N}\right)$ on $\mathcal{H}_{t+1, N}\left(\bar{S}_{t}\right)$, then for any $\varepsilon>0$ there exists $h_{t+1, N}^{\varepsilon} \in \mathcal{H}_{t+1, N}\left(\bar{S}_{t}\right)$ such that $V_{t}\left(\bar{S}_{t}, h_{t+1, N}^{\varepsilon}\right) \geq V_{t}^{*}\left(\bar{S}_{t}\right)-\varepsilon$. Then we have

$$
\begin{gathered}
V_{t-1}^{*}\left(\bar{S}_{t-1}\right)=\sup _{h_{t, N} \in \mathcal{H}_{t, N}\left(\bar{S}_{t-1}\right)} \inf _{Q \in \mathcal{P}_{t}\left(\bar{S}_{t-1}\right)} \int V_{t}\left(\bar{S}_{t}, h_{t+1, N}\right) d Q \\
=\sup _{H_{t} \in D_{t}\left(\bar{S}_{t-1}\right)} \sup _{h_{t+1, N} \in \mathcal{H}_{t+1, N}\left(\bar{S}_{t}\right)} \inf _{Q \in \mathcal{P}_{t}\left(\bar{S}_{t-1}\right)} \int V_{t}\left(\bar{S}_{t}, h_{t+1, N}\right) d Q \\
\geq \sup _{H_{t} \in D_{t}\left(\bar{S}_{t-1}\right)} \inf _{Q \in \mathcal{P}_{t}\left(\bar{S}_{t-1}\right)} \int V_{t}\left(\bar{S}_{t}, h_{t+1, N}^{\varepsilon}\right) d Q \\
\geq \sup _{H_{t} \in D_{t}\left(\bar{S}_{t-1}\right)} \inf _{Q \in \mathcal{P}_{t}\left(\bar{S}_{t-1}\right)} \int V_{t}^{*}\left(\bar{S}_{t}\right) d Q-\varepsilon .
\end{gathered}
$$


Since (A3) is true for any $\varepsilon>0$, from (A2) and (A3) we get Equation (9).

To prove the second part of the Theorem, note that $H^{*}$ is optimal iff $V_{0}\left(\bar{S}_{0}, H^{*}\right)=V_{0}^{*}\left(\bar{S}_{0}\right)$, which follows from the definition of the value function. Let $h_{t, N}^{*}$ be the tail strategies of $H^{*}$ for $t=1, \ldots, N$. Next, we will prove that

$$
V_{t-1}\left(\bar{S}_{t-1}, h_{t, N}^{*}\right)=V_{t-1}^{*}\left(\bar{S}_{t-1}\right), \quad t=1, \ldots, N,
$$

by backward induction. Since $h_{1, N}^{*} \equiv H^{*}$ by definition, it will prove the optimality of $H^{*}$. For $t=N$, (A4) follows from (9) and the definition of $V_{t-1}\left(\bar{S}_{t-1}, h_{t, N}\right)$. Assume that $V_{t}\left(\bar{S}_{t}, h_{t+1, N}^{*}\right)=V_{t}^{*}\left(\bar{S}_{t}\right)$ for some $t \leq N$. Let $\bar{S}_{t}^{*}$ be the combination of the history $\bar{S}_{t-1}$ and the state $S_{t}^{*}$, induced by $H_{t}^{*}$. Then from (A1) we get that

$$
\begin{gathered}
V_{t-1}\left(\bar{S}_{t-1}, h_{t, N}^{*}\right)=\inf _{Q \in \mathcal{P}_{t}\left(\bar{S}_{t-1}\right)} \int V_{t}\left(\bar{S}_{t}^{*}, h_{t+1, N}^{*}\right) d Q=\inf _{Q \in \mathcal{P}_{t}\left(\bar{S}_{t-1}\right)} \int V_{t}^{*}\left(\bar{S}_{t}^{*}\right) d Q \\
=\sup _{H_{t} \in D_{t}\left(\bar{S}_{t-1}\right)} \inf _{Q \in \mathcal{P}_{t}\left(\bar{S}_{t-1}\right)} \int V_{t}^{*}\left(\bar{S}_{t}\right) d Q=V_{t-1}^{*}\left(\bar{S}_{t-1}\right),
\end{gathered}
$$

which proves the induction step and, consequently, the second part of the Theorem.

\section{Proof of Lemma 1}

Proof. The first statement follows from Theorem 3.2 of Reference [27] and implies that the infimum of $\int f(x) Q(d x)$ over $\mathcal{P}_{t}(\cdot)$ equals the infimum of $\sum_{i=1}^{n+1} p_{i} f\left(x_{i}\right)$ over $x_{i} \in K_{t}(\cdot)$ and $p_{i} \geq 0, i=1, \ldots, n+1$, such that $\sum_{i=1}^{n+1} p_{i} x_{i}=E_{t}(\cdot)$ and $\sum_{i=1}^{n+1} p_{i}=1$. The second part can be proven by using the first statement. From the Krein-Milman theorem [28] and the concavity of $f(x)$, we get that the infimum over measures concentrated at $n+1$ or fewer points of $K_{t}(\cdot)$ is greater or equal than the infimum over measures concentrated at $(n+1)^{2}$ or fewer extreme points of $K_{t}(\cdot)$, that is the infimum of $\sum_{i=1}^{(n+1)^{2}} p_{i}^{\prime} f\left(x_{i}^{\prime}\right)$ over $x_{i}^{\prime} \in \operatorname{ext}\left(K_{t}(\cdot)\right)$ and $p_{i}^{\prime} \geq 0, i=1, \ldots,(n+1)^{2}$, such that $\sum_{i=1}^{(n+1)^{2}} p_{i}^{\prime} x_{i}^{\prime}=E_{t}(\cdot)$ and $\sum_{i=1}^{(n+1)^{2}} p_{i}^{\prime}=1$. For any given $x_{1}^{\prime}, \ldots, x_{(n+1)^{2}}^{\prime} \in \operatorname{ext}\left(K_{t}(\cdot)\right)$, this problem is a linear programming problem in the standard form, which is known to have a basic feasible solution $p_{1}^{*}, \ldots, p_{(n+1)^{2}}^{*}$ with $n+1$ or fewer nonzero elements. Therefore, the infimum actually coincides with the infimum over measures concentrated at $n+1$ or fewer extreme points of $K_{t}(\cdot)$, which proves the result.

\section{Proof of Theorem 3}

Proof. The statement is proven by backward induction. For $t=N$, it holds by the assumptions about $J(\cdot)$. Let the subfunctions $\bar{H}_{t}, \bar{W}_{t}^{Y} \mapsto V_{t}^{*}\left(\bar{S}_{t}\right)$ be concave and let the subfunctions $\bar{W}_{t}^{Y} \mapsto V_{t}^{*}\left(\bar{S}_{t}\right)$ be non-decreasing for some $t \leq N$.

Consider the function

$$
v_{t}\left(\bar{H}_{t-1}, \bar{X}_{t-1}, \bar{W}_{t-1}^{Y}, H_{t}, \xi\right)=V_{t}^{*}\left(\bar{H}_{t-1}, \bar{X}_{t-1}, \bar{W}_{t-1}^{Y}, H_{t}, \Lambda(\xi) X_{t-1}, W_{t}^{Y}\left(\bar{H}_{t-1}, \bar{X}_{t-1}, \bar{W}_{t-1}^{Y}, H_{t}\right)\right) .
$$

Note that the subfunctions $\bar{H}_{t}, \bar{W}_{t-1}^{Y} \mapsto W_{t}^{Y}\left(\bar{S}_{t-1}, H_{t}\right)$ are concave and the subfunctions $\bar{W}_{t-1}^{Y} \mapsto W_{t}^{Y}\left(\bar{S}_{t-1}, H_{t}\right)$ are non-decreasing due to the assumptions about $C_{t}\left(\bar{S}_{t-1}, H_{t}\right)$ and the definition (4) of $W_{t}^{Y}\left(\bar{S}_{t-1}, H\right)$. This, when combined with the concavity and monotonicity of $V_{t}^{*}$, implies that the subfunctions $\bar{H}_{t}, \bar{W}_{t-1}^{Y} \mapsto v_{t}\left(\bar{H}_{t-1}, \bar{X}_{t-1}, \bar{W}_{t-1}^{Y}, H_{t}, \xi\right)$ are concave and that the subfunctions $\bar{W}_{t-1}^{Y} \mapsto v_{t}\left(\bar{H}_{t-1}, \bar{X}_{t-1}, \bar{W}_{t-1}^{Y}, H_{t}, \xi\right)$ are non-decreasing. Therefore, the subfunctions 
$\bar{H}_{t}, \bar{W}_{t-1}^{Y} \mapsto \int v_{t}\left(\bar{H}_{t-1}, \bar{X}_{t-1}, \bar{W}_{t-1}^{Y}, H_{t}, \xi\right) Q(d \xi)$ are concave and the subfunctions $\bar{W}_{t-1}^{Y} \mapsto$ $\int v_{t}\left(\bar{H}_{t-1}, \bar{X}_{t-1}, \bar{W}_{t-1}^{Y}, H_{t}, \xi\right) Q(d \xi)$ are non-decreasing by the properties of the integral. Consider

$$
\phi_{t}\left(\bar{S}_{t-1}, H_{t}\right)=\inf _{Q \in \mathcal{P}_{t}\left(\bar{S}_{t-1}\right)} \int v_{t}\left(\bar{H}_{t-1}, \bar{X}_{t-1}, \bar{W}_{t-1}^{Y}, H_{t}, \xi\right) Q(d \xi) .
$$

The subfunctions $\bar{H}_{t}, \bar{W}_{t-1}^{Y} \mapsto \phi_{t}\left(\bar{S}_{t-1}, H_{t}\right)$ are concave as the infima of the concave functions, and the subfunctions $\bar{W}_{t-1}^{Y} \mapsto \phi_{t}\left(\bar{S}_{t-1}, H_{t}\right)$ are non-decreasing due to the monotonicity of $\mathcal{P}_{t}$ and the properties of infimum.

By definition, $V_{t-1}^{*}\left(\bar{S}_{t-1}\right)=\sup _{H_{t} \in D_{t}\left(\bar{S}_{t-1}\right)} \phi_{t}\left(\bar{S}_{t-1}, H_{t}\right)$. For any admissible price history $\bar{X}_{t-1}$ and any $\varepsilon>0$, consider the admissible risky volume histories $\bar{H}_{t-1}^{1}, \bar{H}_{t-1}^{2}$ and risk-free value histories $\bar{W}_{t-1}^{Y, 1}, \bar{W}_{t-1}^{Y, 2}$. By the definition of supremum, there exist $H_{t}^{1} \in D_{t}\left(\bar{H}_{t-1}^{1}, \bar{X}_{t-1}, \bar{W}_{t-1}^{Y, 1}\right)$ and $H_{t}^{2} \in D_{t}\left(\bar{H}_{t-1}^{2}, \bar{X}_{t-1}, \bar{W}_{t-1}^{Y, 2}\right)$ such that

$$
\begin{aligned}
& \phi_{t}\left(\bar{H}_{t-1}^{1}, \bar{X}_{t-1}, \bar{W}_{t-1}^{Y, 1}, H_{t}^{1}\right) \geq V_{t-1}^{*}\left(\bar{H}_{t-1}^{1}, \bar{X}_{t-1}, \bar{W}_{t-1}^{Y, 1}\right)-\varepsilon, \\
& \phi_{t}\left(\bar{H}_{t-1}^{2}, \bar{X}_{t-1}, \bar{W}_{t-1}^{Y, 2}, H_{t}^{2}\right) \geq V_{t-1}^{*}\left(\bar{H}_{t-1}^{2}, \bar{X}_{t-1}, \bar{W}_{t-1}^{Y, 2}\right)-\varepsilon .
\end{aligned}
$$

Consider some $\alpha_{1}, \alpha_{2} \geq 0, \alpha_{1}+\alpha_{2}=1$. From the concavity of $\phi_{t}$, we get that

$$
\begin{gathered}
V_{t-1}^{*}\left(\alpha_{1} \bar{H}_{t-1}^{1}+\alpha_{2} \bar{H}_{t-1}^{2}, \bar{X}_{t-1}, \alpha_{1} \bar{W}_{t-1}^{Y, 1}+\alpha_{2} \bar{W}_{t-1}^{Y, 2}\right) \\
\geq \phi\left(\alpha_{1} \bar{H}_{t-1}^{1}+\alpha_{2} \bar{H}_{t-1}^{2}, \bar{X}_{t-1}, \alpha_{1} \bar{W}_{t-1}^{Y, 1}+\alpha_{2} \bar{W}_{t-1}^{Y, 2}, \alpha_{1} H_{t}^{1}+\alpha_{2} H_{t}^{2}\right) \\
\geq \alpha_{1} \phi\left(\bar{H}_{t-1}^{1}, \bar{X}_{t-1}, \bar{W}_{t-1}^{Y, 1}, H_{t}^{1}\right)+\alpha_{1} \phi\left(\bar{H}_{t-1}^{2}, \bar{X}_{t-1}, \bar{W}_{t-1}^{Y, 2}, H_{t}^{2}\right) \\
\geq \alpha_{1} V_{t-1}^{*}\left(\bar{H}_{t-1}^{1}, \bar{X}_{t-1}, \bar{W}_{t-1}^{Y, 1}\right)+\alpha_{2} V_{t-1}^{*}\left(\bar{H}_{t-1}^{2}, \bar{X}_{t-1}, \bar{W}_{t-1}^{Y, 2}\right)-\varepsilon .
\end{gathered}
$$

Since the above inequality holds for any $\varepsilon>0$, the subfunctions $\bar{H}_{t-1}, \bar{W}_{t-1}^{Y} \mapsto V_{t-1}^{*}\left(\bar{S}_{t-1}\right)$ are concave.

To prove the monotonicity of $\bar{W}_{t}^{Y} \mapsto V_{t}^{*}\left(\bar{S}_{t}\right)$, for any admissible price history $\bar{X}_{t-1}$ and risky volume history $\bar{H}_{t-1}$, consider the admissible risk-free value histories $\bar{W}_{t-1}^{Y, 1}$ and $\bar{W}_{t-1}^{Y, 2}$, such that $\bar{W}_{t-1}^{Y, 1} \leq \bar{W}_{t-1}^{Y, 2}$. Then, $D_{t}\left(\bar{H}_{t-1}, \bar{X}_{t-1}, \bar{W}_{t-1}^{Y, 1}\right) \subseteq D_{t}\left(\bar{H}_{t-1}, \bar{X}_{t-1}, \bar{W}_{t-1}^{Y, 2}\right)$ due to the monotonicity of $D_{t}(\cdot)$, and we use the monotonicity of $\bar{W}_{t-1}^{Y} \mapsto \phi_{t}\left(\bar{S}_{t-1}, H_{t}\right)$ to get that

$$
\begin{aligned}
& V_{t-1}^{*}\left(\bar{H}_{t-1}, \bar{X}_{t-1}, \bar{W}_{t-1}^{Y, 1}\right)=\sup _{H_{t} \in D_{t}\left(\bar{H}_{t-1}, \bar{X}_{t-1}, \bar{W}_{t-1}^{Y, 1}\right)} \phi_{t}\left(\bar{H}_{t-1}, \bar{X}_{t-1}, \bar{W}_{t-1}^{Y, 1}, H_{t}\right) \\
\leq & \sup _{H_{t} \in D_{t}\left(\bar{H}_{t-1}, \bar{X}_{t-1}, \bar{W}_{t-1}^{Y, 2}\right)} \phi_{t}\left(\bar{H}_{t-1}, \bar{X}_{t-1}, \bar{W}_{t-1}^{Y, 2}, H_{t}\right)=V_{t-1}^{*}\left(\bar{H}_{t-1}, \bar{X}_{t-1}, \bar{W}_{t-1}^{Y, 2}\right),
\end{aligned}
$$

which proves that the subfunctions $\bar{W}_{t-1}^{Y} \mapsto V_{t-1}^{*}\left(\bar{S}_{t-1}\right)$ are non-decreasing. This concludes the proof of the induction step and proves the statement.

\section{References}

1. Samuelson, P. Lifetime portfolio selection by dynamic stochastic programming. Rev. Econ. Stat. 1969, 51, 239-246. [CrossRef]

2. Merton, R. Lifetime portfolio selection under uncertainty: The continuous-time case. Rev. Econ. Stat. 1969, 51, 247-257. [CrossRef]

3. Karatzas, I.; Shreve, S. Methods of Mathematical Finance; Springer: Berlin/Heidelberg, Germany, $1998 ;$ p. 415.

4. Magill, M.; Constantinides, G. Portfolio selection and transactions costs. J. Econ. Theory 1976, 13, $245-263$. [CrossRef] 
5. Davis, M.H.A.; Norman, A.R. Portfolio selection with transaction costs. Math. Oper. Res. 1990, 15, 676-713. [CrossRef]

6. Kabanov, Y.; Safarian, M. Markets with Transaction Costs: Mathematical Theory; Springer Science \& Business Media: Berlin/Heidelberg, Germany, 2009.

7. Bertsimas, D.; Lo, A.W. Optimal control of execution costs. J. Financ. Mark. 1998, 1, 1-50. [CrossRef]

8. Almgren, R.; Chriss, N. Value under liquidation. Risk 1999, 12, 61-63.

9. Almgren, R. Optimal execution with nonlinear impact functions and trading-enhanced risk. Appl. Math. Financ. 2003, 10, 18. [CrossRef]

10. Jorion, P. Risk management lessons from Long-Term Capital Management. Eur. Financ. Manag. 2000, 6, 277-300. [CrossRef]

11. Obizhaeva, A.; Wang, J. Optimal trading strategy and supply/demand dynamics. J. Financ. Mark. 2013, 16, 1-32. [CrossRef]

12. Framstad, N.; Øksendal, B.; Sulem, A. Optimal consumption and portfolio in a jump diffusion market with proportional transaction costs. J. Math. Econ. 2001, 35, 233-257. [CrossRef]

13. Smirnov, S.N. Guaranteed deterministic approach to superhedging: Market model, trading constraints and Bellman-Isaacs equations. Math. Game Theory Appl. 2018, 10, 59-99.

14. Smirnov, S.N. Guaranteed deterministic approach to superhedging: "No arbitrage" properties of the market. Math. Game Theory Appl. 2019, 11, 68-95.

15. Deng, X.T.; Li, Z.F.; Wang, S.Y. A minimax portfolio selection strategy with equilibrium. Eur. J. Oper. Res. 2005, 166, 278-292. [CrossRef]

16. Ben-Tal, A.; Margalit, T.; Nemirovski, A. Robust modeling of multi-stage portfolio problems. In High Performance Optimization; Springer: Berlin/Heidelberg, Germany, 2000; pp. 303-328.

17. Talay, D.; Zheng, Z. Worst case model risk management. Financ. Stoch. 2002, 6, 517-537. [CrossRef]

18. Glasserman, P.; Xu, X. Robust risk measurement and model risk. Quant. Financ. 2014, 14, 29-58. [CrossRef]

19. Ellsberg, D. Risk, ambiguity, and the Savage axioms. Q. J. Econ. 1961, 75, 643-669. [CrossRef]

20. Föllmer, H.; Schied, A. Stochastic Finance: An Introduction in Discrete Time; Walter de Gruyter: Berlin, Germany, 2004.

21. Epstein, L.G.; Schneider, M. Recursive multiple-priors. J. Econ. Theory 2003, 113, 1-31. [CrossRef]

22. Iyengar, G.N. Robust Dynamic Programming. Math. Oper. Res. 2005, 30, 257-280. [CrossRef]

23. González-Trejo, J.; Hernández-Lerma, O.; Hoyos-Reyes, L.F. Minimax control of discrete-time stochastic systems. SIAM J. Control Optim. 2002, 41, 1626-1659. [CrossRef]

24. Smirnov, S.N. A Guaranteed Deterministic Approach to Superhedging: The Case of Convex Payoff Functions on Options. Mathematics 2019, under review.

25. Schied, A.; Schöneborn, T. Risk aversion and the dynamics of optimal liquidation strategies in illiquid markets. Financ. Stoch. 2009, 13, 181-204. [CrossRef]

26. Vath, V.L.; Mnif, M.; Pham, H. A model of optimal portfolio selection under liquidity risk and price impact. Financ. Stoch. 2007, 11, 51-90. [CrossRef]

27. Winkler, G. Extreme points of moment sets. Math. Oper. Res. 1988, 13, 581-587. [CrossRef]

28. Krein, M.; Milman, D. On extreme points of regular convex sets. Stud. Math. 1940, 9, 133-138. [CrossRef]

(C) 2019 by the author. Licensee MDPI, Basel, Switzerland. This article is an open access article distributed under the terms and conditions of the Creative Commons Attribution (CC BY) license (http://creativecommons.org/licenses/by/4.0/). 